\title{
Empirical Negation
}

\author{
Michael De
}

Received: 7 June 2011 /Accepted: 22 November 2011 /Published online: 7 February 2012

(C) The Author(s) 2012. This article is published with open access at Springerlink.com

\begin{abstract}
An extension of intuitionism to empirical discourse, a project most seriously taken up by Dummett and Tennant, requires an empirical negation whose strength lies somewhere between classical negation ('It is unwarranted that. . . ') and intuitionistic negation ('It is refutable that. . .'). I put forward one plausible candidate that compares favorably to some others that have been propounded in the literature. A tableau calculus is presented and shown to be strongly complete.
\end{abstract}

Keywords Intuitionistic negation · Empirical negation · Strong negation · Verificationism · Dummett

\section{Introduction}

In mathematical discourse a uniform treatment of negated and unnegated statements can be given by defining the former in terms of the latter. If $A$ is a mathematical statement then its negation may be defined as $A \rightarrow \perp$ where $\perp$ is either taken as primitive or as an abbreviation of some fixed absurdity such as $0=1$. What counts as an "absurdity" will depend on the background formal theory. Notice that in the usual arithmetical setting, defining the negation of $A$ as $A \rightarrow 0=1$ gives us the constructive properties of negation we expect. For example, ex falso quodlibet - that from $A$ and $\neg A$ anything follows - is derivable from modus ponens and the fact that $0=1$ implies everything. Negation introduction and elimination are then just special cases of implication introduction and elimination.

However, there are issues concerning the choice of absurdity. If a theory does not contain sufficient arithmetic then $0=1$ may not do. Indeed, there may be no single sentence able to play the role of absurdity across all mathematical discourses. Instead we shall have to choose, for a given mathematical theory, some sentence able to assume the role of absurdity. In practice this poses no problem on the assumption-

\footnotetext{
M. De $(\square)$

Department of Philosophy, Utrecht University, Janskerkhof 13A, room 1.10, 3512 BL Utrecht, Netherlands

e-mail: mikejde@gmail.com
} 
and this assumption seems safe enough - that we can effectively choose a suitable absurdity for any given theory we are likely to care about. For example in arithmetic we may choose $0=1$, in the theory of strict linear orders $0<0$, and so on.

Constructively there is nothing problematic about implication interpreted according to the familiar BHK clauses nor of an absurdity such as $0=1$, so negation turns out unproblematic in mathematical discourse. ${ }^{1}$ Is the same true for empirical discourse? Dummett thinks not, stating:

Negation . . . is highly problematic. In mathematics, given the meaning of "if . . . then", it is trivial to explain "Not A" as meaning "If A, then $0=1$ "; by contrast, a satisfactory explanation of "not", as applied to empirical statements for which bivalence is not, in general, taken as holding, is very difficult to arrive at. Given that the sentential operators cannot be thought of as explained by means of the two-valued truth-tables, the possibility that the laws of classical logic will fail is evidently open: but it is far from evident that the correct logical laws will always be the intuitionistic ones. More generally, it is by no means easy to determine what should serve as the analogue, for empirical statements, of the notion of proof as it figures in intuitionist semantics for mathematical statements. (Dummett 1996, p.473)

A blanket term for the analogue of proof for empirical statements is warrant or verification. One need not spell out a precise theory of warrant in formulating a semantics whose primary semantic values are warrants. Indeed, there has been no precise spelling out of proof for the constructivist, since proof for them is taken as intuitive and not relative to a given formal theory. Of course this has not prevented the formulation of numerous semantics for constructive logics. ${ }^{2}$ A similar point is made by Kleene regarding the realizability interpretation of intuitionistic number theory when he states " $[t]$ he analysis which leads to this truth definition is not to be regarded as more than a partial analysis of the intuitionistic meaning of the statements, since it takes over without analysis, or leaves unanalyzed, the component of evidence" (Kleene 1945, p. 110).

In what follows I shall be taking the notion of warrant or verification as primitive and assuming, moreover, that a naïve extension of a constructively acceptable semantics to empirical discourse is one which replaces proofs as semantic values with warrants (perhaps with other necessary modifications made as well). ${ }^{3,4}$ The

\footnotetext{
${ }^{1}$ It should be mentioned in passing that some (e.g. (Cook and Cogburn 2000)) have objected to the use of $0=1$ in a definition of negation. I shall not enter into this debate here.

${ }^{2}$ Taking proof as proof-in-L, for some fixed logic L, has been thought to be problematic for constructivism for reasons having to do with Gödel's incompleteness theorems. They would seem to imply that, if constructive proof were just proof-in-L, there would be verification- or proof-transcendent truths, contra constructivism as it is typically conceived. See e.g. Martin-Löf's (Martin-Löf 1984, p. 11).

${ }^{3}$ Williamson (Williamson 1994) makes this suggestion regarding the BHK clauses in order to show that a semantics so extended cannot make sense of empirical statements of the form 'A may be undecided'. I briefly discuss his argument in section 7.

${ }^{4}$ While I have taken the notion of warrant as primitive, so that one may fill in their favorite theory of warrant in the discussion to follow, that discussion does highlight what basic properties warrants must have. For instance, in a constructivist setting the notion of warrant is regarded as monotonic in the sense that if A is warranted by a particular state $\mathrm{a}$, and $\mathrm{b}$ stands in the relevant "inclusion" relation to a then $\mathrm{A}$ is warranted also at $b$.
} 
verification of a statement $\mathrm{A}$ might be taken to be the holding in principle of sufficient empirical evidence in support of A, where what counts as evidence is either contextually determined or domain specific. For instance, seemings to John might count as evidence for 'John is hungry' whereas they may not, e.g. because John has a fever, for 'The temperature is above twenty degrees celcius'. In other words, a number of factors may serve to determine what counts as evidence for a given class of propositions. In the case of mathematics, proof then turns out to be a species of warrant. ${ }^{5}$

It is easy to see why the usual notion of constructive negation does not by itself suffice for expressing negation in empirical discourse. Suppose we wish to express that Goldbach's conjecture is undecided at present. According to the arrow-falsum definition, this statement is equivalent to 'If Goldbach's conjecture is decided (i.e. proved or refuted) at present, then $0=1$ '. But this is far too strong: it states that, with respect to any evidential state we may be in, that Goldbach's conjecture is decided is refutable. ${ }^{6}$ However, while it is vacuously true with respect to our present evidential state that a proof of Goldbach's conjecture - which we do not presently have - can be transformed into a proof of $0=1$, this may not be true with respect to future evidential states at which the conjecture may turn out proved or refuted. To express such claims the constructivist needs a weak negation that, when appended to a statement, expresses that the statement lacks warrant at this evidential state, the state at present. Such a negation has been referred to as 'empirical' in (Williamson 1994)) and (DeVidi and Solomon 2006) and as 'factual' antecedently in (Heyting 1971, p. 18).

It follows that any negation stronger than intuitionistic negation will be too strong to express that a particular evidential state (e.g. the present one) fails to support a given statement. For if the negation is stronger than intuitionistic negation it will express at least that the statement fails to be supported in every extension of the given state. In particular then, strong negation cannot serve as an empirical negation in our intended sense, but not for the reasons Williamson (Williamson 1994) cites. In (Williamson 1994) Williamson suggests, but ultimately rejects, the use of any nonstandard intuitionistic negation, and in particular strong negation, as an empirical negation. I discuss his argument in $\$ 7$.

Dummett proposes that for empirical discourse we treat verification and falsification on a par by taking them as sui generis notions. However, if we do so then we must give up a uniform treatment of the conditions under which a sentence is verified or falsified. The reason is that there is not a fixed (decidable, atomic) statement A to serve as the absurdity which follows from an arbitrary empirical falsehood and from which empirical negation could be defined as implication to A. On this, Dummett remarks:

[w]e might regard the meanings of negations of numerical equations as being given directly in terms of the computation procedures by which those equations

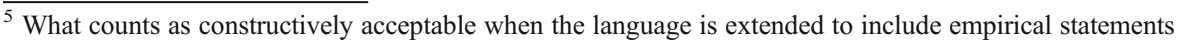
is unclear. For instance it is not clear that disjunction ought to satisfy the disjunction property, that if $\mathrm{A} \vee \mathrm{B}$ is warranted (recalling that the A and B may be empirical) then either A is or B is. This issue is raised again in section 4 but even more needs to be said concerning constructive acceptability.

${ }^{6} \mathrm{I}$ am assuming that if a statement is intuitionistically refutable with respect to some evidential state $\mathrm{s}$, it is refutable with respect to all (or at least all extensions of s), i.e. refutability is not a relative matter.
} 
are verified or falsified: a proof of the negation of any arbitrary statement then consists of an effective method for transforming any proof of that statement into a proof of some false numerical equation. Such an explanation relies on the underlying presumption that, given a proof of a false numerical equation, we can construct a proof of any statement whatsoever. It is not obvious that, when we extend these conceptions to empirical statements, there exists any class of decidable atomic statements for which a similar presumption holds good; and it is therefore not obvious that we have, for the general case, any similar uniform way of explaining negation for arbitrary statements. It would therefore remain well within the spirit of a theory of meaning of this type that we should regard the meaning of each statement as being given by the simultaneous provision of a means for recognizing a verification of it and a means for recognizing a falsification of it, where the only general requirement is that these should be specified in such a way as to make it impossible for any statement to be both verified and falsified. (Dummett 1996, pp. 71-72)

A semantics which treats verification and falsification symmetrically along the lines just sketched by Dummett has been proposed by Thomason (Thomason 1969) for the strong negation of (Nelson 1949). Similarly, Gurevich (Gurevich 1977) motivates his semantics for strong negation by observing that "[i]n many cases the falsehood of a simple scientific sentence can be ascertained as directly (or indirectly) as its truth. An example: a litmus-paper is used to verify sentence [sic] "The solution is acid" "((Gurevich 1977, p. 49)) by which he means 'verify the falsity of' when he says 'verify'. In the context of constructivism we see that symmetrical treatments of truth and falsity have been around for quite some time.

How should an empirical negation behave? If we take the following quote of Dummett seriously then, at least relative the class of statements we have in mind, empirical negation should look nearly classical:

Our reluctance to say that pi was not transcendental before 1882 , or, more generally, to construe mathematical statements as significantly tensed, is not merely a lingering effect of platonistic misconceptions; it is, rather, to speak in this way would be to admit into mathematical statements a non-intuitionistic form of negation, as will be apparent if one attempts to assign a truth-value to 'pi is not algebraic', considered as a statement made in 1881. This is not because the 'not' which occurs in '. . . is not true' or '. . . was not true' is non-constructive: we may reasonably view it as decidable whether or not a statement has been proved at a given time. But though constructive, this is an empirical type of negation that occurs in statements of intuitionistic mathematics. (Dummett 1977, p. 337, my emphasis)

If it is decidable whether or not a given statement A lacks warrant at present (or any given time more generally), it will always be true, for example, that 'either $\mathrm{A}$ is (now) warranted or it is not'.

Empirical negation cannot simply be classical negation if we wish to add it in a straightforward way to the usual proof systems for intuitionistic logic. For there is no straightforward way of introducing classical negation to intuitionistic logic without 
the two negations collapsing into classical negation. ${ }^{7}$ So on pain of collapse, empirical negation must forgo certain classical principles. The same issue arises within the setting of classical relevant logic (see (Meyer and Routley 1973) and (Meyer and Routley 1974)), where the familiar law of contraposition in "arrow-form" fails (though it holds in rule form) for "classical" negation, again, on pain of collapse. Precisely which principles our account of empirical negation forgoes is discussed in Section 4.

However, besides collapse, there is a host of other objections against classical and classical-like negations that any proposal for a constructively or relevantly acceptable negation will have to avoid. In the next section I look at these objections in turn and argue that none of them are a problem for the empirical negation to be introduced in Section 3, despite its close affinity to classical negation. Indeed the following section could serve double duty as a survey of the numerous objections to classical-like negations.

\section{Constructivist Objections to Classical (and like) Negations}

\subsection{Conservativity}

If $\mathbf{L}$ and $\mathbf{L}^{\prime}$ are logics in the respective languages $\mathcal{L}$ and $\mathcal{L}^{\prime}$ with $\mathcal{L} \subseteq \mathcal{L}^{\prime}$, then $\mathbf{L}^{\prime}$ is a conservative extension of $\mathbf{L}$ when for every sentence $A$ in $\mathbf{L}$, if $\mathbf{A}$ is $\mathbf{L}^{\prime}$-provable then it is L-provable. Conservativity has been thought to be a necessary condition on meaning coherence in the sense that the rules governing a connective can only confer coherent meaning on that connective if they conservatively extend a given coherent base. ${ }^{8}$ There are a number of reasons for desiring conservativity having to do primarily with anti-holism, learnability, anti-realism and consistency (against e.g. tonk-like connectives), but whichever reasons one has in mind, classical negation is going to be problematic since the usual ways of proof-theoretically extending deductive systems for intuitionistic logic to include classical negation yield nonconservative extensions. A famous example witnessing this nonconservativity phenomenon (assuming that the meaning of $\rightarrow$ is determined by the usual arrow introduction and elimination rules) is famously Peirce's law, $((A \rightarrow B) \rightarrow A) \rightarrow A$, which is classically but not intuitionistically provable. As such, classical negation has been deemed incoherent, most notably by Dummett.

We need not enter the debate about whether conservativity is a necessary condition for coherence, since one can remain neutral on the issue in cases where a given connective meets the constraint. The empirical negation introduced in Section 3 yields a conservative extension to intuitionistic logic, and so failure of conservativity is no objection to it.

\footnotetext{
$\overline{7}$ There are ways of introducing classical negation without collapse, see e.g. [del Cerro and Herzig 1996], but they are not straightforward adaptations of the usual style of proofs systems (e.g. Hilbert or sequent calculi). ${ }^{8}$ Dummett is often thought to have held the conservativity constraint. There are a number of criticisms of the constraint which seem to settle the matter against it. One is that there may be two connectives each of which can be individually and conservatively added to a given logic though the addition of both yields a nonconservative extension. Is each of these connectives individually coherent until present together? That seems an implausible thing to say.
} 


\subsection{Harmony}

Inferentialists, who hold that the meaning of certain expressions is determined completely by the rules which govern them, have been the initial defenders of conservativity. In the case of the logical connectives they hold that the introduction and elimination rules (sometimes just those of one type and not of the other) completely determine the meaning of a logical connective. ${ }^{9}$ But if conservativity fails as a requirement for coherence, the inferentialist will need to appeal to some other requirement to rule out classical negation as incoherent. Harmony has been thought to fill this role. Roughly, harmony is the constraint that introduction and elimination rules for a logical connective must be in harmony in the sense that the elimination rules warrant precisely what is warranted from the premises of the introduction rules alone. ${ }^{10}$ Some inferentialists have defended harmony while rejecting conservativity (e.g. see (Read 2000)). Inferentialism is typically restricted to the view that the meaning of the logical fragment of our vocabulary is given completely by inferential rules. This is sometimes referred to as moderate inferentialism. One might wish to extend inferentialism to language as a whole, but it is unlikely that an advocate of any such program would endorse harmony as a meaning-theoretic constraint since it is far too constraining.

Should we require of empirical negation that it be governed by harmonious introduction-elimination rules? If empirical negation is non-logical and we are moderate inferentialists, then the answer is clearly "No". On the other hand, if empirical negation is deemed logical on grounds of topic neutrality, then the familiar intuitionistic negation cannot be deemed logical on the same grounds and this seems implausible. For recall that empirical negation is an operation on warrants of which proofs are a special case, so in this sense it generalizes intuitionistic negation by applying to a broader class of propositions, the mathematical and empirical. Now it is unlikely that any inferentialist will accept this brief argument denying the logicality of intuitionistic negation on grounds of topic neutrality, in which case they must hold that, while topic neutrality may be a sufficient condition on logicality, it cannot be a necessary one. According to an inferentialist, being characterizable by rules satisfying certain proof-theoretic constraints (e.g. harmony, conservativity, etc.) will be necessary and sufficient for logicality. But whatever conditions end up being necessary and sufficient for logicality, it is dubious that empirical negation will turn out logical on those grounds. Thus the issue of harmony seems irrelevant to empirical negation. ${ }^{11}$

This is not to say empirical negation fails to satisfy proof-theoretic constraints necessary and sufficient for logicality. Indeed one might hold that the burden of proof

\footnotetext{
${ }^{9}$ Indeed, it has been suggested that an operator is logical when its meaning is determined completely by its rules of inference.

${ }^{10}$ Thanks to an anonymous referee for clarifying some important points concerning my formulation of harmony. The one given here corresponds to what Dummett (Dummett 1991, ch. 13) calls 'stability'.

${ }^{11}$ From a semantic perspective, empirical negation likely will not be counted logical either. For instance, relative to Kripke semantics, empirical negation is non-logical according to the criterion of permutation invariance which states that a connective is logical if the set of truths in a model having that connective as primary is invariant under permutations of the set of worlds, where truth in a Kripke model may be taken to be truth at every or some distinguished point.
} 
lies on the opponent to show that empirical negation cannot be given harmonious rules, which is an extremely strong claim to establish. But it is also debatable whether the rules given in Section 5 for empirical negation are not harmonious. Read (Read 2008) argues that labeled natural deduction systems for a variety of normal modal logics provide a way of furnishing harmonious rules for intensional connectives, and if he is right then empirical negation, and a good deal of other connectives including non-logical ones, satisfy the harmony constraint. ${ }^{12}$

So far the objections to classical and like negations have been proof theoretic, but there is a long history of model-theoretic objections as well. A number of them have been voiced by relevantists in defending non-classical (paraconsistent) De Morgan negation as an alternative to classical negation. Exactly these objections are open also to the intuitionist. In the following section I will discuss these objections in their original relevantist formulations and show how they connect directly to the present topic of empirical negation.

\subsection{Heredity}

Meyer and Routley have shown that relevant logic, once touted as a fierce rival to classical logic, can be viewed rather as an extension to classical logic with additional intensional (relevant) implication and (De Morgan) negation operators. One need only collapse the ordering $\leq$ on states, defined by

$$
a \leq b \text { iff } R 0 a b
$$

for 0 the "base" state, by requiring that $a \leq b$ iff $a=b .{ }^{13}$ Collapsing the ordering to identity is required in order to ensure that the Heredity condition,

$$
\text { (Heredity) If } M, a \vDash A \text { and } a \leq b \text { then } M, b \vDash A \text {, }
$$

is preserved when defining boolean negation $\sim$ by $^{14}$

$$
M, a \vDash \sim A \text { iff } M, a \not A .
$$

The formal semantics of relevant logic has taken a fair amount of criticism from its inception for not having an adequate informal interpretation and the collapse of the ordering that allows for boolean negation worsens that criticism. ${ }^{15}$ For the ordering is supposed to represent an intuitive notion corresponding to something like information gathering, so that $a \leq b$ when $\mathrm{b}$ is an informational extension of $a$. The restriction

\footnotetext{
${ }^{12}$ The use of labeled systems makes Read's approach controversial. The proof language makes explicit use of an "accessibility" relation and formula labels typically thought of corresponding to points in a Kripke structure. In fact, there are rules specifically for the accessibility relation. The question, then, is whether such proof languages presume a prior understanding of the Kripke semantics from which they arose and whether they are thereby anti-inferentialist in spirit.

${ }^{13}$ A model for relevant logic is a quadruple $(W, 0, R, V)$ where $W$ is a non-emtpy set, $0 \in W, R$ is a ternary relation on $W$ and $V$ is a function from $\mathrm{W}$ to subsets of $W$.

${ }^{14}$ The literature on relevant logic refers to the negation defined by (1) as 'boolean' rather than 'classical', so I have stuck with this terminology. In a sense boolean negation is a natural characterization of classical negation for relational semantics, but I don't think it is the only natural one. Fixed negation seems to me to be just as natural.

${ }^{15}$ B. J. Copeland has been a main critic of relevantist semantics, charging them with being merely "pure" rather than "applied". See e.g. (Copeland 1979), (Copeland 1983) and (Copeland 1986).
} 
requiring that $a \leq b$ iff $a=b$ claims that states have no proper extensions and embodies the unfounded Leibnizian optimism that each state is itself the best (in terms of informational content) amongst all possible ones.

The point is that states are not perfect-i.e. complete and consistent. Moreover propositions, construed as sets of states, are not just any such sets: they are the hereditary ones. Now it is clear that boolean negation defined according to (1) is not an operation on propositions in the sense that the class of propositions is closed under that operation. For there are states $a$ and $b$ with $a \leq b$ such that $a$ does not support a proposition $A$, and hence supports its boolean negation, while $b$ supports $A$. But then the boolean negation of $A$ is not preserved $\leq$-upward, and so the boolean negation of A is not a hereditary set (read 'proposition'). As boolean negation is just set-theoretic complementation in disguise, another way of putting this is to say that complementation is not an operation on propositions.

Notice that the same objection may be levelled against classical negation by any intuitionist who takes seriously a semantics on which boolean negation fails to be an operation on propositions intuitionistically conceived. In Kripke semantics for intuitionistic logic (see Section 3), propositions, being $\leq$-closed (for $\leq$ a preorder), are not closed under boolean negation. That is, in a Kripke model $M$ for intuitionistic logic we may have $M, a \vDash \sim A$ and yet $M, b \not \sim A$ (equivalently, $M, b \vDash A$ ) for some $b \geq a$. Now the intuitionist might attempt the same move as the relevantist by collapsing the preorder to identity (obtaining the class of "sheer reflexive" frames) so that Heredity is preserved in the presence of boolean negation, but that will not work since the resulting logic is classical (i.e. the set of arguments valid on the class of sheer reflexive frames is classical logic). So the relevantist move is unavailable to the intuitionist who must instead reject boolean negation as a genuine propositional operation.

DeVidi and Solomon (DeVidi and Solomon 2006) work around this problem by introducing an empirical negation, defined in the context of Kripke semantics, that satisfies Heredity. Roughly the idea is this. The intuitionistic negation $\neg A$ of $A$ is supported at a state iff $A$ is not supported at any ( $\leq-)$ later state. We might think of some subset of states as having a property special to empirical negation, call this property being "actualized" (as put in (DeVidi and Solomon 2006)) at some time, and define negation relative to this property. Then we might think the empirical negation $\sim A$ of $A$ is supported at a state when it is not supported at any later state that is actualized. To be precise, let $M=(W, A, \leq, V)$ be a usual Kripke model for intuitionistic logic with $A \subseteq \mathrm{W}$ an additional set of actualized states. Define the truth conditions for $\sim$ by

1. $M, a \models \sim A$ iff $\forall b$, if $a \leq b$ and $b \in A$ then $b \not A$.

It is easy to see that Heredity holds for the language extended with $\sim$, that is, $\sim$ is a genuine propositional operation.

There are two serious problems with this "negation". The first is that it is too weak. Almost no properties thought characteristic of negation hold for it. In particular, the law of excluded middle, a property we should think holds for empirical negation if we follow Dummett's remark quoted earlier, fails as does the law of non-contradiction and various directions of the De Morgan equivalences (e.g. $\sim(A \wedge B) \mid=\sim A \vee \sim B$ ). Second, it does not get things right at the level of models for there will be states 
supporting $\sim A$ for every $A$, i.e. states at which trivialism holds, a thesis rejected by any constructivist. This will be the case, e.g., when no later state is actualized in which case the truth conditions for $\sim A$ will be vacuously satisfied for arbitrary $A$.

For these reasons the empirical negation to be defined in Section 3 is better motivated from a philosophical point of view and it also fairs much better in terms of getting correct the inferences that ought to intuitively hold of empirical negation.

\subsection{Implicit vs Explicit Information}

Relevantists (e.g. Greg Restall) have argued that there is a "difference between claims about states, and claims supported by states" (Restall 1999, p. 71). A state $a$ 's failing to support a proposition A should not imply that a supports some other proposition $\rightarrow$ A expressing $a$ 's lack of support for A-for this other proposition is about $a$. That is, it should not be assumed generally that a state supports all the information about itself. An intuitionist who even only weakly endorses Kripke semantics could file the same charge against empirical negation if introducing it into the semantics requires making the unjustified identification of implicit and explicit information.

Of course some states might support all the information about themselves, but to say that all do is to make an unfair assumption about states. It would, however, be just as unfair to assume that states never support some or all of the information about themselves. Moreover, why not think that every model ought to possess at least one such state intended to represent a privileged state of the model, for example, the present state of available evidence (or warrant)? Such states will be complete in the sense that every statement will be either warranted or not.

Given Dummett's remark claims of the form ' $A$ is warranted' are in principle decidable, we should think that the present state of available evidence either warrants or fails to warrant the assertion of a given proposition. That is, the present state of available evidence is complete relative to empirical negation $\sim$ in the sense that $A \vee \sim A$ holds good at it. In modeling empirical negation, then, we should include at least one state representing the present state of available evidence for any claim of the form $\sim A$ asserts that, at present, $A$ lacks sufficient evidence to be warranted. Such a state will form a classical model in the sense that the set of sentences in the language involving only the connectives $\wedge$ and $\sim$ will be maximally classically-consistent.

\section{The Semantics of Fixed Negation}

Since we will be interpreting a connective $\sim$ to be read 'There is insufficient evidence at present ${ }^{16}$ to warrant the proposition that. . .' ( $\neg$ will continue to be read 'It is refutable that. ..') we must distinguish one state in each model as the present moment. Such a state will be further singled out by the truth conditions given to our empirical negation $\sim$. These informal ideas motivate the semantical clauses for

\footnotetext{
${ }^{16}$ The usual intensional semantics for temporal indexicals such as 'at present' (i.e. 'now') treats such expressions as non-indexical propositional operators, and this is how we have chosen to treat them here. The same is true of non-temporal expressions such as 'actually' which is taken, by e.g. Lewis, to be indexical.
} 
the connectives of our language, to be specified in a moment, the only novel one being that for $\sim$.

Our language $\mathcal{L}^{\sim}$ is a usual language $\mathcal{L}$ for IPC (Intuitionistic Propositional Logic) augmented with the logical symbol $\sim{ }^{17}$ Let $F=(W, \leq)$ be a Kripke frame for the language $\mathcal{L}$ of IPC. That is, $W$ is a non-empty set of states and $\leq$ is a partial order (reflexive, transitive and anti-symmetric relation) on $W$. We denote by $W \uparrow$ the set of all upsets in $W$, i.e. sets $X$ s.t. $y \in X$ whenever $x \in X$ and $x \leq y$. An $\mathcal{L}^{\sim}$-model $M$ is a tuple $(F, @, V)$ where $@ \in W$ is a distinguished element representing the state of available evidence in $M$ (other modally inequivalent states representing substates or states containing evidence unavailable in $M$ ) and $V:$ Prop $\rightarrow W \uparrow$ is a propositional valuation assigning propositions (i.e. upsets in $\mathrm{W}$ ) to propositional letters. ${ }^{18}$

We arrive at the following truth conditions (writing $M, a \mid=A$ to mean $A$ is supported by a state a in the model $M$ ):

2. $M, a \mid=A \wedge B$ iff $M, a \mid=A$ and $M, a \mid=B$;

3. $M, a \mid=A \vee B$ iff $M, a \mid=A$ or $M, a \mid=B$;

4. $M, a \mid \neq \perp$ (i.e it is never the case that $M, a \mid=\perp$ );

5. $M, a \mid=A \rightarrow B$ iff $\forall b \geq a(M, b|=A \Rightarrow M, b|=B)$;

6. $M, a \mid=\sim A$ iff $M, @ \mid \neq A$.

It is easy to verify by induction on formula complexity that (Heredity),

$$
M, a \mid=A \text { and } a \leq b \text { imply } M, b \mid=A,
$$

holds, as in the case of IPC, since $M, a \mid=\sim A$ iff for all $b \in W, M, b \mid=\sim A$. Notice that clause 6 is a special case of clause 1 of (DeVidi and Solomon 2006) when $A=\{@\}$ and each model is assumed to contain@. One may immediately notice the similarity of $\sim$ to the satisfaction operators of hybrid logic, the "actually" operator, and the "now" operator of temporal logics. ${ }^{19}$

Truth in a model is truth at @, validity on a frame is truth in every model based on that frame, and validity "simpliciter" is validity on every frame. We say that a sentence $A$ is an $\mathcal{L}^{\sim}$-consequence of set $\Gamma$ of sentences, in symbols $\Gamma \mid=A$, iff for every model $M, A$ is true in $M$ whenever every member of $\Gamma$ is true in $M$. We denote the set of valid consequences $\{(\Gamma, A): \Gamma \mid=A\}$ by IPC $^{\sim}$.

We might have required that all models be rooted, i.e. that there be a minimum with respect to the partial order, or, perhaps more in line with common informal interpretations of intuitionistic models, that the models be tree-like. In any case, one

\footnotetext{
${ }^{17}$ For definiteness, the set of logical symbols is $\{\wedge, \vee, \rightarrow, \sim, \perp\}$, and Prop is a denumerable set of propositional letters (constants) whose members we denote by $p$, etc. We define $\neg A:=A \rightarrow \perp$.

${ }^{18}$ We may read $\leq$ in a number of different ways. My preferred way is in terms of informational containment so that $a \leq b$ reads ' $b$ contains all of the information contained in $a$ '. Even though $@$, is to be thought of as the present state of evidence, we need not read $\leq$ temporally, e.g. by reading $a \leq b$ as ' $\mathrm{b}$ is a state of evidence arrived at temporally later than a which contains all the information contained in a' for we might just think of each state in the model as representing a possible present state of evidence.

${ }^{19}$ One interesting application of is that it may also be seen as providing an alternative, but inequivalent, characterization of boolean negation in the setting of relevant logic. If we confine ourselves to the simplified semantics for R then we may introduce by letting '@, denote the sole base world. Valid consequence on the simplified semantics for $\mathbf{R}$ is already defined in the same way we have defined valid consequence for IPC $\sim$, viz. in terms of truth preservation at @. It would be interesting to compare $\sim$ and boolean negation in the setting of relevant logic, a curiosity I mention only to set aside.
} 
obtains the same semantic consequence relation so we have chosen not to restrict our models in any of these two ways. It is interesting to note, however, that there are differences immaterial to consequence: e.g. if @ is always the root then $A \vee \sim A$ holds at every point of every model, whereas this is not true otherwise.

There are two notions of truth in a model we may distinguish: (i) actual truth which is truth at the distinguished element, and (ii) global truth which is truth common to all points in the model. A sentence is actually true in $M=(W, \leq, @, V)$ when it is true at @, and a sentence is globally true in $M$ when it is true at every state a $\in W$ of $M$. These notions of truth are obviously quite distinct. We may also distinguish between two types of consequence: local and actual consequence. A sentence $A$ is a local consequence of a set $\Gamma$ of sentences when for each model $M=$ $(W, \leq, @, V)$ and every $b$ in $M$, if every member of $\Gamma$ is true at $\mathrm{b}$ then so is $A$. And $A$ is an actual consequence of $\Gamma$ when $A$ is true at @ whenever every member of $\Gamma$ is. For the base intuitionistic language $\mathcal{L}$, the distinction between actual and local truth is one without a difference. ${ }^{20}$ However, this is not true for $\mathcal{L}^{\sim}$ since now the distinguished element plays a significant role, where before it did not.

I wish to quickly settle a possible objection with the proposed semantics. Hossack writes

[i]f a sign is to be regarded a negation of $p$ at all, it must be used in such a way as to be incompatible with the assertion that $p$. The semantic rule has to hold, which Dummett calls Exclusion, that $p$ and its negation cannot both be true. (Hossack 1990, p. 216).

One possible objection to the semantics for negation introduced here is that we have states at which both $A$ and $\sim A$ are supported in apparent violation of Dummett's Exclusion condition. For instance let $M=(W, \leq, V)$ be the two element model with $W=\{@, a\}, \leq$ the reflexive closure of $\{\langle @, a\rangle\}$ and $V(p)=\{a\}$. Then $M$, $a \mid=A \wedge \sim A$. But what is crucial here is that it is never the case, in accordance with Exclusion, that both $A$ and $\sim A$ are true, for truth (relative to an IPC $^{\sim}$-model) is truth at @. Notice that the empirical negation of (DeVidi and Solomon 2006) fails to satisfy the Exclusion condition and so would not be deemed a negation according to Hossack and Dummett.

Before moving onto the next section, it is worth discussing an issue that has been raised concerning monotonicity and warrant. Tomassi (Tomassi 2006) states:

...non-monotonicity naturally suggests itself as a logical characteristic definitive of [defeasible warrant]. At least, it is difficult to see how warrant could genuinely be defeasible if it is not the case that further information could be obtained to defeat an assertion so warranted. To allow that defeating information can turn up however is precisely to allow the possibility that while $\mathrm{X}$ defeasibly warrants $\mathrm{Z}$, the conjunction of $\mathrm{X}$ and $\mathrm{Y}$ might warrant the negation of $\mathrm{Z}$. . there is no obvious way of

\footnotetext{
${ }^{20}$ A proof showing local validity implies actual validity is easy; the converse is not much harder. $\mathcal{L}$ models are $\mathcal{L}^{\sim}$-models and the truth conditions for $\mathcal{L}$-sentences are the same as those for $\mathcal{L}^{\sim}$-sentences restricted to $\mathcal{L}$, where $\mathcal{L} \subset \mathcal{L}^{\sim}$. For reductio, suppose $A$ is an actual but not local consequence of $\Gamma$. Then there is a model $M=(W, \leq, @, V)$ and $a \in M$ s.t. $M \mid=\Gamma$ but $M, a \mid \neq A$. But then the model $M^{\prime}=\left(W, \leq, @{ }^{\prime}\right.$, $V$ ), where $@=$, is s.t. $M^{\prime}, @ \mid=\Gamma$ and $M^{\prime} @ \mid \neq A$, which contradicts our supposition that $A$ is an actual consequence of $\Gamma$.
} 
capturing the non-monotonic character of defeasible warrant within the framework provided by Kripke semantics for intuitionist logic. (Tomassi 2006, p.37-38)

While I agree that defeasible warrant introduces an element of non-monotonicity, I do not think it manifests at the level of logical consequence. Defeasible warrant is nonmonotonic over the progression of time relative to some partial ordering on states of evidence. That is, if $\mathrm{a}$ and $\mathrm{b}$ are states of evidence such that $a \leq b$, where $\leq$ is some monotonic ordering of strength of evidence, and $\mathrm{A}$ is warranted at $a$, then $\mathrm{A}$ is warranted at $\mathrm{b}$. What we do not have is that if $\mathrm{A}$ is warranted at some time $t$ and $t<u$ then $\mathrm{A}$ is warranted at $\mathrm{u}$. One has to distinguish between the orderings over which empirical truth is preserved. A statement $\mathrm{A}$ is warranted, in our sense, at a particular time $\mathrm{t}$ if and only if all the available evidence at $\mathrm{t}$ warrants $\mathrm{A}$; i.e. statements are warranted relative to bodies of evidence. We should not want statements to be warranted relative to sets of statements, as they would be if reasoning with a defeasible warrant operator were non-monotonic. If we know that $\mathrm{A}, \vdash \mathrm{B}$ but $\mathrm{A}, \mathrm{C} \vdash \mathrm{B}$, and both $\mathrm{A}$ and $\mathrm{C}$ are warranted, then $\mathrm{B}$ is not warranted relative to $\{A\}-$ it is simply not warranted. Ideally one should distinguish in the model two separate orderings, one $\leq$ over evidence and another $\preccurlyeq$ over temporal states, and introduce a future or past temporal operator $\square$ into the language governed by $\preccurlyeq$ such that A may hold according to some body of evidence at a moment $t$ even though $\square$ A may not hold according to that same body of evidence and moment because $\mathrm{A}$ fails at the relevant $\preccurlyeq$-successors of $\mathrm{t}$.

\section{Validities and Invalidities}

The following lists some validities and inferences involving $\sim$ and shows a significant number of similarities between empirical and classical negation, most notably the holding by the former of all of the De Morgan equivalences, DNE, LEM and LNC, rule-form (EFQ), and classical reductio ad absurdum (RAA).

$$
\begin{aligned}
& A \vee \sim A \\
& \sim \sim A \rightarrow A \\
& (\sim A \rightarrow A) \rightarrow A \\
& \neg A \rightarrow \sim A \\
& \sim(A \wedge B) \leftrightarrow(\sim A \vee \sim B) \\
& \sim(A \vee B) \leftrightarrow(\sim A \wedge \sim B) \\
& \sim(A \rightarrow B) \rightarrow \sim B \\
& \neg \sim A \rightarrow A \\
& (A \vee \sim B) \rightarrow \sim(A \rightarrow B) \\
& A \wedge \sim A \mid=B \\
& \sim(A \wedge \sim A) \\
& A \rightarrow B \mid=\sim B \rightarrow \sim A
\end{aligned}
$$

The following lists some notable exclusions to the above list, in particular all of the $\rightarrow$-forms of contraposition.

$$
\begin{array}{ll}
(\sim A \rightarrow \sim B) \rightarrow(B \rightarrow A) & (A \rightarrow B) \rightarrow(\sim B \rightarrow \sim A) \\
(A \wedge \sim A) \rightarrow B & \sim(A \rightarrow B) \rightarrow(A \wedge \sim B) \\
\neg(A \wedge \sim A) & \sim A \rightarrow \neg A
\end{array}
$$

Nearly all of the rule-forms of contraposition fail as well, a feature familiar to relevant logicians who have observed these sorts of failures for boolean negation in the setting of classical relevant logic. 
It is worth noting that $\mathbf{I P C}^{\sim}$ is not closed under substitution of provable equivalents, though it is obviously closed under uniform substitution. A logic is closed under substitution of provable equivalents when $\Gamma \vdash A, B \vdash C$ and $C \vdash B$ imply $\Gamma \vdash A(B / C)$, where $A(B / C)$ is the result of replacing some occurrences of $B$ in $A$ with occurrences of $C$. A logic is closed under uniform substitution when $\Gamma \vdash A$ implies $\Gamma \vdash A(p / q)$ for $p, q$ atoms. For example $p \wedge \sim p \vdash p \wedge \neg p$ and $p \wedge \neg p \vdash p \wedge \sim p$, but $\vdash(p \wedge \neg p) \rightarrow \perp$ while $\vdash(p \wedge \sim p) \rightarrow \perp$. This is not a particularly uncommon phenomenon. For example, compare logics with "actually" operators ${ }^{21}$, temporal logics with "now" operators, and paraconsistent logics with non-truth-functional negations such as those of de Costa 1974, each of which fails to be closed under substitution of provable equivalents.

More importantly, $A \mid=B$ is weaker than $\mid=A \rightarrow B$ since the latter implies the former but not conversely - in other words, conditional proof fails. This explains, e.g., the $\rightarrow$-form failure of EFQ; $\rightarrow$ may take us to states at which both $A$ and $\sim A$ hold, and hence states where an arbitrary $A$ (e.g. $\perp$ ) need not (or in the case of the example, must not) hold. Often it is the implication from $\mid=A \rightarrow B$ to $A \mid=B$ that fails. For instance, this is the case with Tennant's relevant logics $\mathbf{C R}$ and IR (see (Tennant 1997)). In particular, $\mid=(A \wedge \neg A) \rightarrow B$ holds but $A, \neg A \mid \neq B$ which is quite the opposite from IPC ${ }^{\sim}$.

It appears the rules for $\rightarrow$ are no longer in harmony since the usual form of $\rightarrow$-elimination (in sequent form, $A \rightarrow B, A \vdash B$ ) holds while $\rightarrow-$ introduction in its usual form (from $\Gamma, A \vdash B$ infer $\Gamma \vdash A \rightarrow B$ ) fails. ${ }^{22}$ But $\rightarrow$-introduction is supposed to be (and is on proposed ways of making rigorous harmony) the unique rule harmonious with $\rightarrow$-elimination. There are two things to say here. First, the elimination rule for $\rightarrow$ in the proof system for IPC $\sim$ of Section 5 is not the usual $\rightarrow$-elimination rule and, moreover, it is harmonious with the introduction rule given in Section 5 in the sense of (Read 2008). There may be other worries concerning the proof system - in particular, whether its use of labels can be justified on inferentialist grounds - that is independent of issues regarding harmony. I must ignore these issues here. Second, it is essential to the failure of $\rightarrow$-introduction that there be occurrences of $\sim$ which means that the usual harmonious rules can be given to $\rightarrow$ for the $\rightarrow$-fragment of IPC $^{\sim}$, whatever consolation that may count as. This is not always the case, e.g. with non-conservative extensions which introduce theorems no longer provable by the original harmonious rules of the base language.

\subsection{Remarks}

Given the failure of conditional proof, IPC $\sim$ is not an axiomatic extension of any finite axiomatization of IPC in the sense that the former is obtainable from the latter by the addition of axioms. For any axiomatic extension of a finitely axiomatized system satisfying conditional proof, i.e. the deduction theorem in the context of

\footnotetext{
$\overline{{ }^{21} \text { For instance, see [Hodes, 1984] }}$ and his notion of "weak" consequence according to which $A$ and $@ A$, the latter read 'actually A', are provably equivalent but not intersubstitutable salva veritate.

${ }^{22}$ Thanks to a referee for pointing this out. As remarked below, harmonious rules can be given to all connectives with respect to a labelled proof system, but without unlabelled system for IPC $\sim$, it cannot be said whether the same is true of other styles of proof systems (e.g. unlabelled natural deduction). This is an interesting open problem.
} 
axiomatic systems, satisfies conditional proof. However, IPC $\sim$ is a conservative extension of IPC. This is easy to see semantically, a self-evidence not usually exuded on the proof-theoretic side. For if $A$ does not contain $\sim$ then it receives the truth conditions it has in IPC-models and so if it were IPC ${ }^{\sim}$-valid then it would have already been IPC-valid. As a final remark we note that IPC $^{\sim}$ is Tarskian in that it satisfies the structural rules of Reflexivity, Thinning and Cut.

Digression: It should not be surprising that the disjunction property, if $\Gamma \vdash A \vee B$ then $\Gamma \vdash A$ or $\Gamma \vdash B$, fails for IPC ${ }^{\sim}$. This sort of failure is notorious for extensions of IPC. (So notorious in fact that it was conjectured by Lukasiewicz that IPC is the strongest intermediate logic to have the disjunction property. ${ }^{23}$ Kreisel and Putnam proved this conjecture false by showing that the system obtained by adding $(\neg A \rightarrow(B \vee C)) \rightarrow((\neg A \rightarrow B) \vee(\neg A \rightarrow C))$ to IPC has the disjunction property. $)$ However, given our intended interpretation of the language it is not hard to justify the failure of conditional proof and the disjunction property. The justification for the failure of the latter rests on the assumption that it is decidable whether or not a proposition is supported by the evidence available at present in which case we expect $\vdash A \vee \sim A$ for arbitrary $A$ without either of $\vdash A$ or $\vdash \sim A$ holding. So we focus our attention on the failure of conditional proof. End of digression.

There are two things to say about the failure of conditional proof. The first is that if we are to take seriously Kripke semantics for intuitionistic logic and if adding empirical negation is semantically acceptable then there is reason to reject conditional proof. After all, it falls out of an acceptable semantics for the language. Now most intuitionists do not find Kripke semantics acceptable primarily on the grounds that it is usually couched within a classical metatheory. But at the propositional level, this should not matter since IPC and its extension $\mathbf{I P C}^{\sim}$ to fixed negation are complete with respect to a recursive set of finitely recursive models (i.e. models with a finite set of states and a recursive accessibility relation and valuation defined on that set). Indeed, if $\Gamma H_{\text {IPC }} A$ then, via a filtration technique, one can construct a finite countermodel from the set of subformulae of $\Gamma \cup\{A\}$ (assuming $\Gamma$ is finite). Restricting the class of models so, the metatheory being classical or intuitionistic then come to the same thing.

While this fact does not generalize to quantificational theories, one may still furnish such theories with a constructive metatheory employing Kripke models even though certain results classically provable will not be provable in the constructive metatheory. This will not be problematic for a constructivist who finds something appealing with an informal interpretation of Kripke structures for intuitionistic logic and who wishes to make use of those structures in providing a semantics for her constructive language while sticking to a constructively acceptable metatheory. I shall therefore suppose that there are constructively acceptable versions of Kripke semantics for intuitionistic logic.

Despite the failure of conditional proof for $\rightarrow$, we may introduce other implication connectives satisfying conditional proof. One natural way to do this is to define $A$ as being a consequence from $\Gamma$, where $\Gamma$ is finite, just when $\mid=\bigwedge \Gamma \rightarrow$

\footnotetext{
${ }^{23}$ Technically, IPC $\sim$ is not an intermediate logic since its language is not $L$ but a proper extension of it. It might be more appropriate to call it a 'superintuitionistic' logic though some authors use these terms synonymously.
} 
A. (Alternatively we could allow infinite sets of premises and infinitary conjunction, but this is unnecessary given compactness for IPC $^{\sim}$.) Under this definition of consequence, consequence and implication coincide but the definition looks ad hoc. Moreover we lose important theorems that were motivated by our original concerns. For example EFQ for $\sim$ fails (since now $\perp$ would not be a consequence of $A \wedge \sim A$ ) as do a number of other important consequences involving the conditional that were valid under the original definition. So the revised definition of consequence does not speak well to the informal interpretation we had originally given to the language.

A more interesting conditional that satisfies conditional proof exploits the fact that (the theory of)@ is essentially a classical model with an additional intensional connective $\rightarrow$. Notice that all the classical connectives are definable at $@$ in a straightforward way, e.g. $A \supset B:=\sim A \rightarrow \sim \sim B$ (or equivalently $\sim A \vee \sim \sim B$ ) says that at an arbitrary point, either $A$ fails to be @. Then $\Gamma \vdash A$ (under the original definition of $\vdash$ ) iff $\vdash \wedge \Gamma \supset A$ for finite $\Gamma$. One with a strong liking for conditional proof might then think of material implication as the correct notion of implication for empirical discourse.

\section{Tableaux}

Several complications present themselves on the side of proof theory. The first, which we have already encountered, is that conditional proof fails. This means that in the setting of natural deduction, $\mathrm{a} \rightarrow$-introduction rule would, in our case, turn out quite cumbersome. (This of course is not true for any system for which conditional proof fails.) The second, more troubling, difficulty is that since $A, \sim A \mid=\perp$ which, in a natural deduction setting, we might call $\sim E$, and $\neg A \mid=\sim A$, it follows that any of the usual presentations of natural deduction would generate a consequence relation satisfying both $\neg A \vdash \sim A$ and $\sim A \vdash \neg A$ (the latter following by $\sim E$ and $\neg I$ ) which is clearly bad (in respect of the latter); for $\sim$ is supposed to be strictly weaker than $\neg$. Exactly the same problem emerges when adding classical negation to intuitionistic logic in the usual systems of natural deduction.

For this reason we have chosen to give a signed and labelled tableau system for IPC $^{\sim}$. The system is standard for IPC for this style of tableau system and adds two additional rules for sentences of the form $S \sim A$ where $S$ is a sign in $\{\mathrm{T}, \mathrm{F}\}$ representing the truth or falsity of a sentence at a state (e.g. 'T $A \wedge B$, i' expresses that $A \wedge B$ is true at state $i$ ). For tableaux there are two types of sentences.

\section{Definition 5.1}

- A signed labelled sentence has the form $S A, i$ for $S \in\{\mathrm{T}, \mathrm{F}\}, A$ an $\mathbf{I P C}^{\sim}$-sentence and $i \in\{@\} \cup \mathrm{N}=$ Labels (the set of labels).

- A relational sentence has the form $i \leq j$ for $i, j \in$ Labels.

A tableau for an argument $A_{1}, \ldots A_{n} \Rightarrow B$, with $A_{\mathrm{i}}$ the premises and $B$ the conclusion, starts by listing the TA, @ in their natural order proceeded by FB,@. More precisely we have the following definition of a tableau. 


\section{Definition 5.2 (Tableaux)}

- A tableau $\mathcal{T}=(\mathcal{X}, \leq)$ is a partially ordered, finite set of sentences (labelled or relational) with a minimum (root) such that for each $\alpha \in \mathcal{T}$ the set of predecessors of $\alpha$ under $\leq$ forms a linear order. If $\leq$ is a partial order on a set $X$ then $\alpha$ is a minimum in $X$ w.r.t. $\leq$ iff for all $\beta \in X, \alpha \leq \beta$.

- A branch of a tableau $\mathcal{T}$ is a maximal chain in $\mathcal{T}$.

\section{Definition 5.3 (Closure)}

- A branch closes iff there is an $i \in$ Labels and sentence $A$ such that both $\mathrm{T} A, \mathrm{i}$ and $\mathrm{F} A, i$ occur on the branch.

- A tableau closes iff each of its branches closes.

- A branch (tree) is open if it is not closed.

A closed tableau for an argument is a proof of that argument. An argument $A_{1}, \ldots$ $A_{n} \Rightarrow B$ is provable when there exists a closed tableau for it, in which case we write $A_{1}, \ldots A_{n} \vdash_{\text {IPC }} \sim B$, sometimes suppressing the subscript when it is clear.

We have the following rules, a pair for each connective $\otimes$ indicating how to decompose sentences preceded by $\mathrm{T}$ or by an $\mathrm{F}$ whose main connective is $\otimes$.

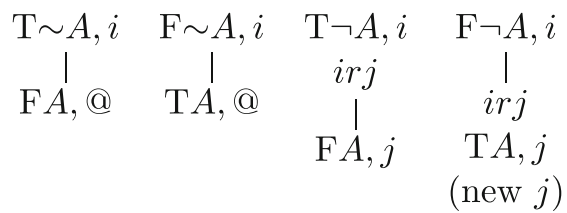

$\mathrm{T} A \wedge B, i \quad \mathrm{~F} A \wedge B, i$

$\mathrm{T} A, i$

$\mathrm{T} B, i$

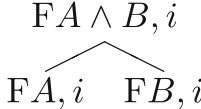
F $A$,

$\mathrm{FB}$

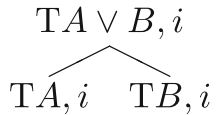

$\mathrm{T} A$

$$
\mathrm{T} A \rightarrow B, i
$$

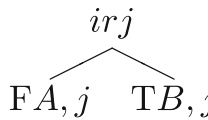
$\mathrm{T} B, j$ $i r j$ $\mathrm{T} A, j$ $\mathrm{F} B, j$ (new $j$ )

The parenthetical '(new $j$ )' indicates that the label $j$ must be new to the branch; i.e. it must not occur as the label of any formula on the branch besides those introduced by the rule. In the second to last rule of the second row, governing the transitivity of $r$, jrk does not need to immediately follow irj on the branch. All that is required is that each occur on the branch. The same may be said about the conclusions of the other rules, though it need not be. For definiteness we understand the other rules to be read in the same light.

\section{Completeness}

The proof of completeness for the tableau system is given according to the usual recipe. We confine ourselves to systematic tableaux, which means that the rules are to be taken as "musts" rather than "cans": if a rule can be applied, it must be applied. A 
systematic tableau is one in which every branch $\beta$ is downward saturated (defined below). The goal then is to show that every open branch of a systematic tableau forms a Hintikka set (defined below), and that every Hintikka set is satisfiable. Completeness then follows almost immediately.

Definition 6.1 A branch $\beta$ is downward saturated provided the following hold.

7. If $\mathrm{T} A \wedge B, i$ occurs on $\beta$ then $\mathrm{T} A, i$ and $\mathrm{T} B, i$ occur on $\beta$.

8. If $\mathrm{T} A \vee B, i$ occurs on $\beta$ then either TA,i occurs on $\beta$ or $\mathrm{T} B, i$ occurs on $\beta$.

9. If $\mathrm{T} \neg A, i$ and irj occurs on $\beta$ then FA, $j$ occurs on $\beta$.

10. If $\mathrm{T} A \rightarrow B, i$ and $i r j$ occurs on $\beta$ then either $\mathrm{F} A, j$ occurs on $\beta$ or $\mathrm{T} A, j$ occurs on $\beta$.

11. If $T \sim A, i$ occurs on $\beta$ then $\mathrm{F} A$, $@$ occurs on $\beta$.

12. If $\mathrm{F} A \wedge B, i$ occurs on $\beta$ then either $\mathrm{F} A, i$ occurs on $\beta$ or $\mathrm{F} B, i$ occurs on $\beta$.

13. If $\mathrm{F} A \vee B, i$ occurs on $\beta$ then both $\mathrm{F} A, i$ and $\mathrm{F} B, i$ occur on $\beta$.

14. If $\mathrm{F} \neg A, i$ occurs on $\beta$ then for some $j, \operatorname{irj}$ and $\mathrm{T} A, j$ occur on $\beta$.

15. If $\mathrm{F} A \rightarrow B, i$ occurs on $\beta$ then for some $j, i r j, \mathrm{~T} A, j$ and $\mathrm{F} B, j$ occur on $\beta$.

16. If $\mathrm{F} \sim A, i$ occurs on $\beta$ then $\mathrm{T} A$, $@$ occurs on $\beta$.

17. For all $i$ occurring on $\beta$, iri occurs on $\beta$.

18. If irj and jrk occur on $\beta$ then irk occurs on $\beta$.

Definition 6.2 A set $H$ of formulas is a Hintikka set if it satisfies the conditions of Definition 6.1 by replacing 'occurs on' by 'is a member of' and ' $\beta$ ' by ' $H$ ', and the further condition that for no propositional letter $p$ and $i \in$ Labels are both $\mathrm{T} p, i$ and $\mathrm{F} p, i$ in $H$.

Lemma 6.3. Every open branch $\beta$ forms a Hintikka set. i.e. the set of formulas consisting of the nodes of $\beta$ is a Hintikka set.

Proof. Immediate by Definitions 5.2 and 6.2.

Lemma 6.4 (Model existence). Every Hintikka set $H$ is satisfiable in an IPC ${ }^{\sim}$-model $M^{H}=(W, \leq, @, V)$ defined as follows. Let label $(H)$ be the set of labels in H, i.e. label $(H)=\{i: S A, i \in H \wedge S \in\{T, F\}\}$. Set

- $\quad W=\operatorname{label}(H)$

- $I \leq j$ iff $i r j \in H$

- @ = @

- $V(p)=\{i \in W: T p, i \in H\}$.

Proof We do two things. The first is to verify that $M^{H}$ is indeed a model. The second is to show that the Fundamental Lemma 6.5 holds.

We claim that $\leq$ is a partial order. Clearly for all $i \in W, i \leq i$ by definition and the fact that for each $i \in$ label $(H)$, iri $\in H$ by the "reflexivity" rule. Transitivity follows similarly by the definition of $\leq$ and the fact that irj, jrk $\in H$ only if $i r k \in H$ by the "transitivity" rule and downward saturation. Anti-symmetry is vacuously satisfied since the rules never allow that $i r j, j r i \in H$ for $i \neq j$. 
To finish verifying that the structure defined above is an IPC ${ }^{\sim}$-model we have only left to show that for no $i \in W$ and $A \in L^{\sim}$ do we have $i \in V(A)$ and $i \in \overline{V(A)}$, where $\bar{X}$ is the $W$-complement of $X$. As the result follows trivially by the definition of $V$ (as the reader may wish to verify), our verification that $M^{H}$ is a model is concluded.

All that is left is proof of the following "Fundamental Lemma".

Lemma 6.5 (Fundamental Lemma). If TA, $i \in H$ then $M^{H}, i \mid=A$, and if FA, $i \in H$ then $M^{H}, i \mid \neq A$.

Proof We proceed by induction on formula complexity.

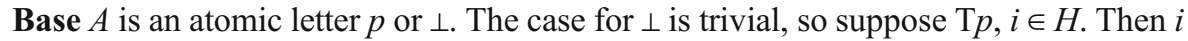
$\in V(p)$, so $M^{H}, i \mid=p$.

Now suppose $\mathrm{F} p, i \in H$. Then $i \notin V(p)$, so $M^{H}, i \mid \neq p$.

Now assume the induction hypothesis (IH) holds for all formulas of complexity less than $A$ 's.

Case $1 A$ is $B \wedge C$. Suppose T $B \wedge C, i \in H$. Then $(\mathrm{T} B, i),(\mathrm{T} C, i) \in H$, so By IH $M^{H}, i$ $\mid=B$ and $M^{H}, i \mid=C$. Hence $M^{H}, i \mid=B \wedge C$.

Now suppose $\mathrm{F} B \wedge C, i \in H$. Then either $\mathrm{F} B, i \in H$ or $\mathrm{F} C, i \in H$. By IH either $M^{H}, i \neq B$ or $M^{H}, i \neq C$ whence $\mathrm{M}^{H}, i \mid \neq B \vee C$

Case $2 A$ is $B \vee C$. Suppose T $B \vee C, i \in H$. Then either T $B, i \in H$ or TC, $i \in H$. By IH either $M^{H}, i \mid=B$ or $M^{H}, i \mid=C$, whence $M^{H}, i \mid=B \vee C$

Now suppose $\mathrm{F} B \vee C, i \in H$. Then both $\mathrm{F} B, i, C, i \in H$. By IH $M^{H}, i \neq B$ and $M^{H}, i \neq B$, whence $M^{H}, i \neq B \vee C$

Case $3 A$ is $\neg B$. Suppose $\mathrm{T} \neg B, i \in H$. Then for all $j$ s.t. $i r j \in H$ and $\mathrm{F} B, j \in H$. By IH, for all $j$ s.t. $i \leq j, M^{H}, j \mid=B$. Hence $M^{H}, i \mid=\neg B$.

Now suppose $\mathrm{F} \neg B, i \in H$. Then there is a $j$ s.t. $\operatorname{irj} \in H$ and $\mathrm{T} B, j \in H$. Then $i \leq j$ and by $\mathrm{IH} M^{H}, j \mid=\mathrm{B}$. Hence $M^{H}, i \mid \neq \neg B$.

Case $4 A$ is $B \rightarrow C$. Suppose T $B \rightarrow C, i \in H$. Then for each $j$ s.t. $i r j \in H$, either $\mathrm{F} B, j$ $\in H$ or TC, $j \in H$. By IH either $M^{H}, j \mid=B$ or $M^{H}, j \mid=C$ for each $j \geq i$. Hence $M^{H}, i \mid=B \rightarrow C$.

Now suppose $\mathrm{F} B \rightarrow C, i \in H$. Then there is a $j$ s.t. $i r j \in H, \mathrm{~T} B, j \in H$ and $\mathrm{F} C, j \in H$. Thus $i \leq j$ and by $\mathrm{IH} M^{H}, j \mid=B$ and $M^{H}, j \neq C$. Hence $M^{H}, i \neq$ $B \rightarrow C$.

Case $5 A$ is $\sim B$. Suppose $\mathrm{T} \sim B, i \in H$. Then $\mathrm{F} B, @ \in H$, whence by $\mathrm{IH} \mathrm{M}^{\mathrm{H}}, @ \neq B$, so $M^{H}, i \mid=\sim \mathrm{B}$.

Now suppose $\mathrm{F} \sim B, i \in H$. Then TB, @ $\in H$, whence by IH $M^{H}, @ \mid=B$, so $\mathrm{M}^{\mathrm{H}}, i \neq \sim B$.

Theorem 6.6 (Completeness). If $\Gamma \vdash B$ then $\Gamma \mid=B$. (Recall that $\Gamma \mid=B$ means that for every model $M$, if $M \mid=\Gamma$ then $M \mid=B$.)

Proof Suppose $\Gamma+\mathrm{B}$. Then there is a systematic tableau $T$ with open branch $\beta$ s.t. for each $\gamma \in \Gamma, \mathrm{T} \gamma, @$ occurs on $\beta$ and FB, @ occurs on $\beta$. Let $H$ be the Hintikka set whose elements are the nodes of $\beta$. Then by the fundamental lemma, there is an IPC ${ }^{\sim}$-model $M^{H}$ s.t. $M^{H}, @ \mid=\Gamma$ and $M^{H}, @ \neq B$, thus $\Gamma \neq B$. Contraposing, if $\Gamma \mid=B$ then $\Gamma \vdash B$. 


\section{Final Remarks}

We have considered an operation $\sim$ such that the truth of $\sim A$ at an arbitrary point of evaluation is determined completely by the truth of $\mathrm{A}$ at a distinguished point representing all evidence available at present. When consequence is defined as preservation of truth in a pointed model, $\sim$ looks nearly classical. Moreover, it seems plausible that any semantics defined with respect to a class of models which distinguishes a point according to which truth in that model amounts to truth at that point, solicits the characterization of a family of connectives defined in terms of the distinguished point.

If verificationism is a theory about ordinary discourse, then any intuitively coherent connective used in such discourse ought to admit of a constructively acceptable semantics where expressive adequacy is a concern. A theory $\mathrm{T}$ is expressively adequate with respect to a connective picked out by natural language expressions if there is a connective $\otimes$ in the language of $T$ which corresponds, in an intuitive sense given the $\vdash_{T}$-rules governing $\otimes$, to $\propto$. For instance, IPC is expressively adequate with respect to conjunction to which $\wedge$ corresponds. IPC is clearly not expressively adequate with respect to weak and empirical negations, just as certain theories of truth (of e.g. Kripke) are not expressively adequate with respect to "exclusion negation" (as opposed to "choice negation"), the operation which takes an intermediate truth value to truth in a three-valued semantics such as Kleene's.

This expressive inadequacy sometimes brings with it predictable paradoxes of an impoverished language as noted e.g. in (Williamson 1994), where Williamson generates a Fitch-like paradox for an intuitionistic language enriched with an additional warrant operator $\mathrm{K}$ read 'at some past, present or future time someone possesses a warrant to assert A'. The argument runs as follows. The claim that $\mathrm{A}$ will never be decided may be formalized as $\neg K A \wedge \neg K \neg A$. But $\neg K A$ implies $\neg A$ (shown below) which, together with $\neg K A \wedge \neg K \neg A$, yields the contradiction $\neg A \wedge \neg \neg A$. Here is the argument Williamson gives, a mild variation of Fitch's original, to show that $\neg K A$ implies $\neg A$.
1. $A \wedge \neg K A$
supposition
2. $K(A \wedge \neg K A) \quad 1$ BHK interpretation
3. $K A \wedge K \neg K A \quad 2$ distribution
4. $K A \wedge \neg K A \quad 3$ axiom $\mathrm{T}$
5. $\neg(A \wedge \neg K A) \quad 1,4 \neg \mathrm{I}$
6. $\neg K A \rightarrow \neg A \quad 5$ intuitionistic propositional logic

If in line (1) we replace $\neg$ with $\sim$ we can obtain everything up to the penultimate line (even with intuitionistic negation out front), but we can not obtain the conclusion, line (6)..$^{24}$

Williamson rejects any solution to this paradox that uses a "non-standard negation" $\rightarrow$ on the grounds that (i) it must satisfy $\rightarrow A \rightarrow \neg A$, and (ii) $K \neg A$ but not $K \neg A$ will have to be treated as sufficient for a negative solution to ' $A$ will one day be decided'. First, his argument for (i) relies on the use of principles that are questionable

\footnotetext{
${ }^{24}$ In fact, the paradox is blocked in a Kripke semantics for IPC with a standard-going S5-like epistemic operator $\mathrm{K}$ since $\mathrm{A} \wedge \neg \mathrm{KA}$ will then be consistent.
} 
on the addition of an empirical negation and $\mathrm{K}$ to the language. One of these is $\neg(A \wedge \neg A)$ that he thinks should hold but which does not in IPC $\sim$. His reason for thinking so is that " $[\neg A]$ should at least be inconsistent with $A$ " (Williamson 1994, 139).

They are inconsistent in IPC $($ when $\rightarrow$ is $\sim$ ) but that need not imply the validity of $\neg(A \wedge \neg A)$ and when the intended discourse is empirical, I see little reason to think it should. Another questionable inference is closure under provable implications, in the form $A \vdash B$ rather than $\vdash A \rightarrow B$, which he makes liberal use of. But this inference may not be warranted in logics extending IPC to include $K$ and $\rightarrow$. Indeed it is not warranted in both IPC $^{\sim}$ and the logic Williamson himself defines on (Williamson 1994, p. 140), a logic of strong negation with a warrant operator.

Williamson's justification of (ii) assumes that the only consistent rendering of 'A will never be decided' for the intuitionist is $\rightarrow(K A \vee K \rightarrow A)$ (for non-standard $\rightarrow$ ), a claim itself established on the assumption of (i), viz. that $\vdash \neg A \rightarrow \neg A$ (see (Williamson 1994, p. 139)). But we have already seen why (i) ought to be rejected and hence (ii) along with it. ${ }^{25}$

Acknowledgements Thanks to Stephen Read, Dick de Jongh, Lloyd Humberstone, Elia Zardini, Ole Hjortland, and Heinrich Wansing for helpful discussion. I would also like to thank a referee of this journal for their substantive and detailed comments, as well as Julia Langkau and the audience of the 3rd Cambridge Graduate Conference on the Philosophy of Logic and Mathematics.

Open Access This article is distributed under the terms of the Creative Commons Attribution License which permits any use, distribution, and reproduction in any medium, provided the original author(s) and the source are credited.

\section{References}

Cook, R. T., \& Cogburn, J. (2000). What negation is not: Intuitionism and $0=1$. Analysis and Metaphysics, $60(1), 5-12$.

Copeland, B. J. (1979). On when a semantics is not a semantics: Some reasons for disliking the routleymeyer semantics for relevance logic. Journal of Philosophical Logic, 8, 399-413.

Copeland, B. J. (1983). Pure semantics and applied semantics. Topoi, 2(2), 197-204.

Copeland, B. J. (1986). What is a semantics for classical negation? Mind, 95(380), 478-490.

del Cerro, L. F. \& Herzig, A. (1996). Combinig classical and intuitionistic logic, or: Intuitionistic implication as a conditional. In Frontiers of Combining Systems (FroCos), pages 93-102

DeVidi, D., \& Solomon, G. (2006). Empirical negation in intuitionisitic logic. In D. DeVidi \& T. Kenyon (Eds.), A Logical Approach to Philosophy (pp. 151-168). Dordrecht, The Netherlands: Springer.

Dummett, M. (1977). Elements of Intuitionism (2nd ed.). USA: Oxford University Press.

Dummett, M. (1991). The logical basis of metaphysics. Harvard University Press

Dummett, M. (1996). The seas of language. Oxford university press.

Gurevich, Y. (1977). Intuitionistic logic with strong negation. Studia Logica, 36(1/2), 49-59.

Heyting, A. (1971). Intuitionism: An Introduction (3rd ed.). Amsterdam: North Holland Publishing Co.

Hodes, H. T. (1984). Some theorems on the expressive limitations of modal languages. Journal of Philosophical Logic, 13, 13-26.

Hossack, K. G. (1990). A problem about the meaning of intuitionist negation. Mind, 99(394), 207-219.

Kleene, S. (1945). On the interpretation of intuitionistic number theory. The Journal of Symbolic Logic, 10 (4), 109-124.

\footnotetext{
${ }^{25}$ This liberates strong negation from Williamson's objection to its use in the paradox he presents. But it does not liberate it from the point made in $\S 1$ that it is too strong to capture that a given statement lacks evidence at a particular state and not necessarily all extensions of that state. For an application of strong negation to Fitch's paradox, see (Wansing, 2002).
} 
Martin-Löf, P. (1984). Intuitionistic Type Theory. Bibliopolis.

Meyer, R. K., \& Routley, R. (1973). Classical relevant logics i. Studia Logica, 32(1), 51-66.

Meyer, R. K., \& Routley, R. (1974). Classical relevant logics ii. Studia Logica, 33(2), 183-194.

Nelson, D. (1949). Constructible falsity. The Journal of Symbolic Logic, 14(1), 16-26.

Read, S. (2000). Harmony and autonomy in classical logic. Journal of Philosophical Logic, 29, $123-154$.

Read, S. (2008). Harmony and modality. In Dégremont, C., Keiff, L., and Rückert, H., editors, Dialogues, Logics and Other Strange Things: Essays in Honour of Shahid Rahman, pages 285-304. College Publications.

Restall, G. (1999). Negation in relevant logics: how i stopped worrying and learned to love the routley star. Applied Logic Series. Kluwer Academic Publishers.

Tennant, N. (1997). The Taming of the True. New York: Oxford University Press.

Thomason, R. (1969). A semantical study of constructible falsity. Zeitschrift für Mathematische Logik und Grundlagen der Mathematik, 15:247-257.

Tomassi, P. (2006). Truth, warrant and superassertibility. Synthese, 148, 31-56.

Wansing, H. (2002). Diamonds are a philosopher's bestfriend. Journal of Philosophical Logic, 31, 591612 .

Williamson, T. (1994). Never say never. Topoi, 13(2), 135-145. 